\title{
COALGEBRAS OVER THE HIGHER RANK SYMPLECTIC GROUPS
}

\author{
ANNA MARIA PAOLUCCI
}

(Communicated by Palle E. T. Jorgensen)

\begin{abstract}
In these notes we generalize the construction obtained for the deformation of the symplectic group $\mathrm{Sp}(2)$ to the case of any $N$ even: $N=2 k$. We characterize the bialgebras $A_{q}(\mathrm{Sp}(k))$ by generators and relations. We consider the deformation of the algebra of polynomials on the group $\mathrm{Sp}(k): \operatorname{Pol}\left(\mathrm{Sp}_{q}(k)\right)$ is a Hopf*-algebra and we build *-representations of it by means of a Verma module construction.
\end{abstract}

\section{INTRODUCTION}

Quantum groups from the algebraic point of view as the spectrum of a Hopf algebra were first introduced by Drinfeld in 1986 [1]. Another different approach is by Jimbo [2] where the quantum groups are considered as the deformations of the universal enveloping algebra of a semisimple Lie algebra. In [3, 12] the term quantum group was introduced by Woronowicz from a different point of view: a quantum group is to be considered as a deformation of the $C^{*}$-algebra of the continuous functions on a compact group. In these notes we will use Woronowicz's approach to quantum groups. Theorem 3.1 gives an explicit characterization of the quantum group $\mathrm{Sp}_{q}(k)$ and it provides the justification of why it is a twisted symplectic group. We consider $\operatorname{Pol}\left(\mathbf{S p}_{q}(k)\right)$ as a deformation of the algebra of polynomials on $\operatorname{Sp}(k)$. It has a structure of a Hopf*-algebra.

We prove a Poincaré-Birkoff-Witt theorem for the bialgebra $A_{q}(\operatorname{Sp}(k))$ and we construct a basis of $\operatorname{Pol}\left(\mathrm{Sp}_{q}(k)\right)$. In $\S 3$ we construct irreducible *-representations by means of a Verma module construction.

\section{NOTATION AND DEFINITIONS}

Let us state the definitions of bialgebra, Hopf algebra, and Hopf *-algebra. Let $A$ be an algebra over $\mathbb{C}$ with unit $I$; we denote the multiplication by

$$
m: A \otimes A \rightarrow A, \quad a \otimes b \rightarrow a b,
$$

and the unit by $\varepsilon: \mathbb{C} \rightarrow A, z \rightarrow z I . A$ is said to be a bialgebra if there exists unital algebra homomorphisms

$$
\varphi: A \rightarrow A \otimes A, \quad e: A \rightarrow \mathbb{C},
$$

Received by the editors September 30, 1992 and, in revised form, January 19, 1993. 1991 Mathematics Subject Classification. Primary 16W30, 17 B37. 
so that

$$
\begin{aligned}
& (\varphi \otimes \mathrm{id}) \varphi=(\mathrm{id} \otimes \varphi) \varphi, \\
& (e \otimes \mathrm{id}) \varphi=\mathrm{id}=(\mathrm{id} \otimes e) \varphi .
\end{aligned}
$$

$\varphi$ is called the comultiplication and $e$ the counit. Property (2.1) is called the coassociativity. The bialgebra $A$ is a Hopf algebra if there exists a linear map $K: A \rightarrow A$ called the antipode such that

$$
(m \circ(K \otimes \mathrm{id}) \circ \varphi)(a)=(\varepsilon \circ e)(a) I=(m \circ(\mathrm{id} \otimes K) \circ \varphi)(a)
$$

for every $a \in A$. We say that $A$ is a Hopf*-algebra if there exists an involutive antilinear mapping $*: A \rightarrow A, a \rightarrow a^{*}$, such that the comultiplication and the counit are *-homomorphisms and $K \circ *$ is involutive, i.e., $K \circ * \circ K \circ *=\mathrm{id}$.

3. The bialgebra $A_{q}(\operatorname{Sp}(k))$ And the Hopf*-Algebra Pol $\left(\operatorname{Sp}_{q}(k)\right)$

In this section we consider the bialgebra $A_{q}(\operatorname{Sp}(k))$ and the Hopf*-algebra $\operatorname{Pol}\left(\operatorname{Sp}_{q}(k)\right)$. We prove a theorem that gives a characterization of $A_{q}(\operatorname{Sp}(k))$ by generators and relations. The bialgebra $A_{q}(\mathrm{Sp}(k))$ may also be viewed as a specialization of the algebra of functions on the $q$-deformations of the symplectic group as defined in [11].

Fix $0<q<1$ and let $A_{q}(\operatorname{Sp}(k))$ be the unital algebra generated by the matrix elements of $u=\left(u_{i j}\right)_{i, j=1, \ldots, N}$, with $N=2 k$. There exist unique unital algebra homomorphisms

$$
\varphi: A_{q}(\operatorname{Sp}(k)) \rightarrow A_{q}(\operatorname{Sp}(k)) \otimes A_{q}(\operatorname{Sp}(k))
$$

and

$$
e: A_{q}(\operatorname{Sp}(k)) \rightarrow \mathbb{C}
$$

so that $e\left(u_{i j}\right)=\delta_{i j}$ and $\varphi\left(u_{i j}\right)=\sum_{k} u_{i k} \otimes u_{k j}$. Then $A_{q}(\operatorname{Sp}(k))$ is a bialgebra with comultiplication $\varphi$ and counit $e$. We define the quantum determinant by

$$
D=\sum_{\sigma \in S_{N}}(-q)^{l(\sigma)} u_{1, \sigma(1)} \cdots u_{N, \sigma(N)},
$$

where $S_{N}$ is the permutation group of $\{1, \ldots, N\}$, and $l(\sigma)$ is the length of the permutation. The quantum minor $D^{i j}$ is defined to be the $(N-1)$ by $(N-1)$ quantum determinant of the matrix obtained from $u$ by deleting the $i$ th row and the $j$ th column. Thus,

$$
D^{i j}=\sum_{\sigma \in S_{N-1}}(-q)^{l(\sigma)} u_{1, \sigma(1)} \cdots u_{i-1, \sigma(i-1)} u_{i+1, \sigma(i+1)} \cdots u_{N, \sigma(N)},
$$

where, indicating by a hat the index to be omitted,

$$
\sigma:\{1, \ldots, \hat{i}, \ldots, N\} \rightarrow\{1, \ldots, \hat{j}, \ldots N\} .
$$

By developing the quantum determinant along a row or a column we have the following equations:

$$
\begin{array}{ll}
\delta_{i j} D=\sum_{k=1}^{N}(-q)^{k-j} u_{i, k} D^{j k}, & \delta_{i j} D=\sum_{k=1}^{N}(-q)^{i-k} D^{k i} u_{k, j}, \\
\delta_{i j} D=\sum_{k=1}^{N}(-q)^{j-k} D^{j k} u_{i, k}, & \delta_{i j} D=\sum_{k=1}^{N}(-q)^{k-i} u_{k, j} D^{k i} .
\end{array}
$$


It is known [11], that in the case of $A_{q}(\mathrm{GL}(N))$ the quantum determinant generates the center of the algebra. We see that $D \in \operatorname{centre}\left(A_{q}(\operatorname{Sp}(k))\right)$. The comultiplication and the counit act on $D$ as follows:

$$
\varphi(D)=D \otimes D, \quad e(D)=1 .
$$

Define the algebra $\operatorname{Pol}\left(\operatorname{Sp}_{q}(k)\right)$ as the extension of $A_{q}(\mathrm{Sp}(k))$, with the element $D^{-1}$ which satisfies

$$
D D^{-1}=I=D^{-1} D .
$$

The comultiplication $\varphi$ and the counit $e$ can be extended to $\operatorname{Pol}\left(\operatorname{Sp}_{q}(k)\right)$ by putting

$$
\varphi\left(D^{-1}\right)=D^{-1} \otimes D^{-1}, \quad e\left(D^{-1}\right)=1 .
$$

We define

$$
K\left(u_{i j}\right)=(-q)^{i-j} D^{j i} D^{-1} \text { and } K\left(D^{-1}\right)=D,
$$

$K$ extends to $\mathrm{Pol}\left(\mathrm{Sp}_{q}(k)\right)$ as a unital linear antimultiplicative mapping. Thus $\operatorname{Pol}\left(\operatorname{Sp}_{q}(k)\right)$ is a Hopf-algebra with comultiplication $\varphi$, counit $e$, and antipode $K$.

We introduce a *-operation on $\operatorname{Pol}\left(\operatorname{Sp}_{q}(k)\right)$ by letting

$$
u_{i j}^{*}=K\left(u_{j i}\right)=(-q)^{j-i} D^{i j} D^{-1} \text { and }\left(D^{-1}\right)^{*}=D .
$$

Thus $D$ is unitary,

$$
D^{*} D=I=D D^{*} \text {. }
$$

So, $\operatorname{Pol}\left(\operatorname{Sp}_{q}(k)\right)$ is a Hopf *-algebra. By (3.1) and (3.2) we have

$$
\sum_{k=1}^{N} u_{i k} u_{j k}^{*}=\delta_{i j} I=\sum_{k=1}^{N} u_{k i}^{*} u_{k j},
$$

i.e., $u$ is unitary.

We now characterize the Hopf *algebra $\operatorname{Pol}\left(\mathrm{Sp}_{q}(k)\right)$ following Woronowicz's definition of quantum group. Let $\left\{e_{1}, \ldots, e_{N}\right\}$ be the standard basis in $\mathbb{C}^{N}$ over $\mathbb{C}$, where $N=2 k$. Let $q$ be a real parameter $0<q<1$; we define the vectors

$$
\xi_{(i)}=e_{i} \otimes e_{k+i}-q e_{k+i} \otimes e_{i}, \quad i=1, \ldots, k .
$$

Thus the $\xi_{i}$ belong to the $\mathbb{C}$-vector space of $q$-alternating 2-tensors $\Lambda^{2}\left(\mathbb{C}^{N}\right)$, for $i=1, \ldots, k$.

Theorem 3.1. Let $y^{\prime}=\left(y_{q} \otimes I\right)$ be the vector belonging to the $\mathbb{C}$-vector space of q-alternating $N$-tensors $\Lambda^{N}\left(\mathbb{C}^{N}\right) \otimes \operatorname{Pol}\left(\operatorname{Sp}_{q}(k)\right)$. Then for any $N$ by $N$ matrix,

$$
u^{*} u=I=u u^{*} \text {, }
$$

with entries $u_{i j}$ belonging to the algebra $\operatorname{Pol}\left(\operatorname{Sp}_{q}(k)\right)$, the following two systems of conditions are equivalent:

(I) The matrix $u=\left(u_{i j}\right)_{i, j=1, \ldots, N}$ satisfies the following conditions:

(a) quantum symplectic invariance condition

$$
(u \otimes I)(I \otimes u) x=x
$$

where $x=\sum_{i=1}^{k} \xi_{i} \otimes \mathrm{id}_{\left.\mathrm{Pol}_{\left(\mathrm{S}_{q}\right.}(k)\right)}$; 
(b) quantum determinant condition

$$
((u \otimes I)(I \otimes u) \otimes \cdots \otimes(u \otimes I)(I \otimes u)) y^{\prime}=y^{\prime}
$$

where the tensor product is repeated $N$ times.

(II) $u$ is of the form

$$
u=\left(\begin{array}{cc}
A & -q B^{*} \\
B & A^{*}
\end{array}\right)
$$

where $A, B$ are $k$ by $k$ matrices with entries in $\operatorname{Pol}\left(\operatorname{Sp}_{q}(k)\right)$, and the $u_{i j}$ satisfy the following relations:

(A) Lie brackets

$$
\left[u_{i j}, u_{l m}\right]=(q-1 / q) u_{l j} u_{i m}, \quad \text { for } 1 \leq i, j \leq k, k+1 \leq l, m \leq N \text {. }
$$

(B) q-relations

(a) $u_{i j} u_{i l}=q u_{i l} u_{i j}$, for $1 \leq i \leq N, 1 \leq j \leq k, k+1 \leq l \leq N$.

(b) Set $\left(z_{i j}\right)=\left(u_{i j}\right)^{T}$, so that $z_{i j}=u_{j i}$; then

$$
z_{i j} z_{i l}=q z_{i l} z_{i j}, \quad \text { for } 1 \leq i \leq N, 1 \leq j \leq k, k+1 \leq l \leq N .
$$

(C) Commutation relations

$$
\begin{aligned}
u_{i j} u_{l m} & =u_{l m} u_{i j}, \quad \text { for } j \geq 1, l \leq k, k+1 \leq i, m \leq N, \\
u_{i i} u_{j j} & =u_{j j} u_{i i}, \quad \text { for } 1 \leq i, j \leq k .
\end{aligned}
$$

(D) Zero divisors

(a)

$$
\begin{aligned}
u_{i j} u_{i+1, l}=0=u_{i+1, l} u_{i j}, \quad \text { for } i & =1, \ldots, N-1, i \text { odd, } \\
1 & \leq j \leq k, k+1 \leq l \leq N, \\
u_{i j} u_{i-1, l}=0=u_{i-1, l} u_{i j}, \quad \text { for } i & =1, \ldots, N, i \text { even, } \\
1 & \leq j \leq k, k+1 \leq l \leq N .
\end{aligned}
$$

(b) Set $\left(z_{i j}\right)=\left(u_{j i}\right)^{T}$, so that $z_{i j}=u_{j i}$; then

$$
\begin{aligned}
z_{i j} z_{i+1, l}=0=z_{i+1, l} z_{i j}, \quad \text { for } i & =1, \ldots, N-1, i \text { odd, } \\
1 & \leq j \leq k, k+1 \leq l \leq N, \\
z_{i j} z_{i-1, l}=0=z_{i-1, l} z_{i j}, \quad \text { for } i & =1, \ldots, N, i \text { even, } \\
1 & \leq j \leq k, k+1 \leq l \leq N .
\end{aligned}
$$

(c)

$$
\begin{array}{ll}
u_{i j} u_{l m}=0=u_{l m} u_{i j}, & \text { for } j \geq 1, l \geq k, 1 \leq i, m \leq k, \\
u_{i j} u_{l m}=0=u_{l m} u_{i j}, & \text { for } j \geq 1, l \geq k, k+1 \leq i, m \leq N .
\end{array}
$$

Proof. (I) $\Rightarrow$ (II). We use the unitarity of the matrix $u$ to simplify the equation (a). Hence we get

$$
(I \otimes u) x=\left(u^{*} \otimes I\right) x .
$$

By rewriting this equation in the matrix form using the basis of $\mathbb{C}^{N} \otimes \mathbb{C}^{N}$ composed of $\left\{e_{i} \otimes e_{j}\right\}$, where $\left\{e_{i}\right\}$ represents the standard basis of $\mathbb{C}^{N}$, we get the desired form of the matrix. 
To prove that (b) gives all the relations between the $u_{i j}$ in (II) we use induction on the dimension $k$. For $N=4$, i.e., $k=2$, it is true in view of Theorem 1 of [6]. Assume then that it is true for some $k \in \mathbb{Z}$. We prove it for $k+1$.

By using the form of the matrix $u$, we can write the following block decomposition obtained from blocks of lower dimensions for which the induction hypothesis applies:

$$
\left(\begin{array}{llll}
A_{0} & a_{1} & B_{0}^{*} & b_{1}^{*} \\
a_{2} & a_{3} & b_{2}^{*} & b_{3}^{*} \\
B_{0} & b_{1} & A_{0}^{*} & a_{1}^{*} \\
b_{2} & b_{3} & a_{2}^{*} & a_{3}^{*}
\end{array}\right) .
$$

Let us see how the induction hypothesis works to give all the relations. Observe that

$$
a_{i}=\left(\begin{array}{c}
a_{i}^{1} \\
\vdots \\
a_{k}^{i}
\end{array}\right), \quad i=1,2,
$$

which is a $k \times 1$ matrix, and similarly

$$
b_{i}=\left(\begin{array}{c}
b_{i}^{1} \\
\vdots \\
b_{k}^{i}
\end{array}\right), \quad i=1,2 .
$$

The zero divisors are obtained by using the induction hypothesis on the $2 \times 2$ matrix whose blocks are

$$
\left(\begin{array}{ll}
A_{0} & a_{1} \\
a_{2} & a_{3}
\end{array}\right), \quad\left(\begin{array}{ll}
B_{0} & b_{1} \\
b_{2} & b_{3}
\end{array}\right), \quad\left(\begin{array}{ll}
A_{0}^{*} & a_{1}^{*} \\
a_{2}^{*} & a_{3}^{*}
\end{array}\right), \quad\left(\begin{array}{ll}
B_{0}^{*} & b_{1}^{*} \\
b_{2}^{*} & b_{3}^{*}
\end{array}\right) .
$$

This implies $A_{0} a_{i}=0, i=1,2,3$, where in this proof this stands for a product of $a_{r s} a_{j}^{i}$, for every $j, r, s, r \neq s$.

We define $a_{i} A_{0} \equiv\left(A_{0}^{*} a_{i}\right)^{*}$, so that we also get $a_{i} A_{0}=0$. In a similar way we proceed to obtain $B_{0} b_{i}=0, i=1,2,3$. For the relations $A_{0} b_{i}^{*}=0, i=$ 2,3 , we proceed as above and obtain the relations by the induction hypothesis; similarly for $b_{i}^{*} A_{0}=\left(A_{0}^{*} b_{i}\right)^{*}=0$. Also,

$$
A_{0} b_{i}=0=b_{i} A_{0}, \quad i=1,3 .
$$

By the induction hypothesis on the matrix of order $2 k$ obtained by suitably deleting rows and columns from the original matrix of order $2 k+2$, the $q$ relations are

$$
A_{0} B_{0}^{*}=q B_{0}^{*} A_{0} .
$$

Similarly by suitably choosing $k \times k$ blocks in the original matrix we have

$$
\begin{gathered}
a_{i} b_{i}^{*}=q b_{i}^{*} a_{i}, \quad i=2,3 . \\
a_{1} B_{0}^{*}=q B_{0}^{*} a_{1} .
\end{gathered}
$$

We proceed in a similar way to verify $A_{0} B_{0}=q B_{0} A_{0}$.

The commutation relations follow by the induction hypothesis in the case $B_{0} B_{0}^{*}=B_{0}^{*} B_{0}$, and by the induction hypothesis and by suitably choosing subblocks for the relations $B_{0} b_{i}^{*}=b_{i}^{*} B_{0}$.

For the Lie bracket relations, to verify that

$$
\left[A_{0}, A_{0}^{*}\right]=\left(q-q^{-1}\right) B_{0} B_{0}^{*},
$$


use the $q$-relations

$$
A_{0} A_{0}^{*}=q B_{0} B_{0}^{*}, \quad A_{0}^{*} A_{0}=q B_{0}^{*} B_{0},
$$

which imply the above Lie brackets relations. On summing the latter formulae, we obtain

$$
A_{0} a_{1}^{*}=q a_{1}^{*} A_{0}, \quad i=1,2,3 .
$$

Hence the result holds.

(II) $\Rightarrow$ (I). This converse implication works in the same way as for the case $n=4$ (see Theorem 1 of [6]).

By considering the form of the matrix $u$, the relations can be equivalently rewritten as

$$
\begin{gathered}
{\left[a_{i j}, a_{k l}^{*}\right]=(q-1 / q) b_{k j} b_{i l}^{*}, 1 \leq i, j \leq k, k+1 \leq l, m \leq N .} \\
a_{i j} b_{i l}^{*}=q b_{i l}^{*} a_{i j}, 1 \leq i \leq N, 1 \leq j \leq k, k+1 \leq l \leq N . \\
a_{j i} b_{l i}=q b_{l i} a_{j i}, 1 \leq i \leq N, 1 \leq j \leq k, k+1 \leq l \leq N . \\
b_{i j} b_{l m}^{*}=b_{l m}^{*} b_{i j}, j \geq 1, l \leq k, k+1 \leq i, m \leq N \\
\left.a_{i i} a_{j j}=a_{j j} a_{i i} \text { (i.e., the diagonal entries of } A\right) .
\end{gathered}
$$

(D1)

$$
\begin{aligned}
& a_{i j} b_{i+1, l}^{*}=0=b_{i+1, l}^{*} a_{i j}, 1 \leq i \leq N-1, i \text { odd, } 1 \leq j \leq k, k+1 \leq l \leq N, \text { or } \\
& b_{i j} a_{i+1, l}^{*}=0=a_{i+1, l}^{*} b_{i j}, 1 \leq i \leq N-1, i \text { odd, } 1 \leq j \leq k, k+1 \leq l \leq N .
\end{aligned}
$$

$$
\begin{aligned}
& a_{i j} b_{i-1, l}^{*}=0=b_{i-1, l}^{*} a_{i j}, 2 \leq i \leq N, i \text { even, } 1 \leq j \leq k, k+1 \leq l \leq N, \text { or } \\
& b_{i j} a_{i-1, l}^{*}=0=a_{i-1, l}^{*} b_{i j}, 2 \leq i \leq N, i \text { even, } 1 \leq j \leq k, k+1 \leq l \leq N .
\end{aligned}
$$

$$
\begin{aligned}
& a_{j i} b_{l, i+1}^{*}=0=b_{l, i+1}^{*} a_{j i}, 1 \leq i \leq N-1, i \text { odd, } 1 \leq j \leq k, k+1 \leq l \leq N, \text { or } \\
& b_{j i} a_{l, i+1}^{*}=0=a_{l, i+1}^{*} b_{j i}, 1 \leq i \leq N-1, i \text { odd, } 1 \leq j \leq k, k+1 \leq l \leq N .
\end{aligned}
$$

$a_{j i} b_{l, i-1}^{*}=0=b_{i-1, l}^{*} a_{j i}, 2 \leq i \leq N, i$ even, $1 \leq j \leq k, k+1 \leq l \leq N$, or $b_{j i} a_{l, i-1}^{*}=0=a_{l, i-1}^{*} b_{j i}, 2 \leq i \leq N, i$ even, $1 \leq j \leq k, k+1 \leq l \leq N$.

$$
\begin{aligned}
& a_{i j} b_{l m}=0=b_{l m} a_{i j} \text { for } j \geq 1, l \geq k, 1 \leq i, m \leq k, \text { or } \\
& a_{i j} a_{l m}=0=a_{l m} a_{i j} \text { for } j \geq 1, l \geq k, 1 \leq i, m \leq k, \text { or } \\
& b_{i j} b_{l m}=0=b_{l m} b_{i j} \text { for } j \geq 1, l \geq k, 1 \leq i, m \leq k . \\
& a_{i j} b_{l m}=0=b_{l m} a_{i j} \text { for } j \geq 1, l \geq k, k+1 \leq i, m \leq N, \text { or } \\
& a_{i j} a_{l m}=0=a_{l m} a_{i j} \text { for } j \geq 1, l \geq k, k+1 \leq i, m \leq N, \text { or } \\
& b_{i j} b_{l m}=0=b_{l m} b_{i j} \text { for } j \geq 1, l \geq k, k+1 \leq i, m \leq N .
\end{aligned}
$$

\section{The Poincare-Birkhoff-Witt theorem AND VERMA MODULE CONSTRUCTION}

We want to construct a basis for the bialgebra $A_{q}(\mathrm{Sp}(k))$ and for the Hopf *-algebra $\operatorname{Pol}\left(\mathrm{Sp}_{q}(k)\right)$ by proving a Poincaré-Birkhoff-Witt theorem for 
$A_{q}(\mathrm{Sp}(k))$. By following [14] we say that if a pair $\left(u_{a b}, u_{c d}\right)$ of matrix elements satisfies relations (A) it is "bad". For a product of matrix elements

$$
x=u_{i_{1} j_{1}} \cdots u_{i_{p} j_{p}}
$$

we define the badness $b(x)$ of $x$ by

$$
b(x)=\#\left\{1 \leq r, s \leq p:\left(u_{i_{r} j_{r}}, u_{i_{s} j_{s}}\right) \text { is bad }\right\} .
$$

We introduce a special ordering on the matrix elements by $u_{i j}<_{0} u_{k l}$ if $i<k$ or if $i=k$ and $j>l$.

Lemma 4.1. For the ordering $<_{0}$ on the elements $u_{i j}$ there is a basis of $A_{q}(\mathrm{Sp}(k))$ consisting of

$$
\left\{u_{i_{1} j_{1}}^{r_{1}} \cdots u_{i_{m} j_{m}}^{r_{m}}: m=N^{2}, r_{1} \in \mathbb{Z}_{+}, u_{i_{1} j_{1}}<_{0} u_{i_{2} j_{2}}<_{0} \cdots<_{0} u_{i_{m} j_{m}}\right\} .
$$

Proof. Set $X=\left\{u_{i j}: 1 \leq i, j \leq N\right\}$. Let $\mathbb{C}\langle X\rangle$ be the free associative algebra generated by $X$. We define a special ordering on the matrix elements by $u_{i j}<_{0} u_{k l}$ if $i<k$ or if $i=k$ and $j>l$. We extend $<_{0}$ to monomials of $\mathbb{C}\langle X\rangle$ by first ordering the degree and for monomials with the same degree by the lexicographical ordering. We introduce a reduction system $S$ for the free associative algebra $\mathbb{C}\langle X\rangle$ as follows. Consider

$$
u=\left(u_{i j}\right)=\left(\begin{array}{cc}
A & -q B^{*} \\
B & A^{*}
\end{array}\right)
$$

as in Theorem 3.1, where $A$ and $B$ are $k$ by $k$ matrices. We now define the operator $L$ by

$L\left(A B^{*}\right)=q B^{*} A$, where for $A B^{*}$ we mean a product of $a_{i j} b_{k l}^{*}$ if $i=k$,

$L\left(A A^{*}\right)=A^{*} A+\left(q-q^{-1}\right) B B^{*}$,

$L(A B)=q B A$, for $a_{i j} b_{k l}$ if $j=l$,

$L(A A \backslash$ diagonal entries $)=0$,

$L(B B)=0$,

$L\left(A B^{*}\right)=0$, for $a_{i j} b_{k l}^{*}$, if $i \neq k$,

$L\left(B B^{*}\right)=B^{*} B$,

$L(A A)=A A$, for diagonal entries,

$L(A B)=0$, for $a_{i j} b_{k l}$, if $j \neq l$.

Let $U=\{A\}, V=\left\{B^{*}\right\}$. Define

$$
\begin{array}{ll}
f_{1}: U \rightarrow V \otimes U, & x \rightarrow 1 \otimes x, \\
f_{2}: V \rightarrow V \otimes U, & y \rightarrow y \otimes 1,
\end{array}
$$

which are well-defined maps. Then there exists a mapping

$$
F: U \times V \rightarrow V \otimes U, \quad F(x, y)=f_{1}(x) f_{2}(y)
$$

by the universality of the tensor product $\tilde{F}: U \otimes V \rightarrow V \otimes U$; set $L=\widetilde{F}$. Hence $L$ is well defined.

The reduction system $S$ is given by $\left\{u_{i j} u_{k l}, L\left(u_{i j} u_{k l}\right)\right\}$, for $u_{1 l}<_{0} u_{i j}$. The ordering is compatible with the reduction system, i.e., for every

$$
x=u_{i j} u_{k l}, \quad L\left(u_{i j} u_{k l}\right)<_{0} u_{i j} u_{k l}
$$


because of the lexicographical ordering for monomials. Every element of $\mathbb{C}\langle X\rangle$ is reduction finite since the number of monomials smaller than a given monomial is finite.

If we prove that all ambiguities are resolvable then one of the equivalent conditions of the diamond lemma [16, Theorem 1.2B] holds. This proves Lemma 4.1. We observe that there are only overlapping ambiguities. Consider

$$
x=u_{r s} u_{k l} u_{i j}
$$

with $u_{r s}<_{0} u_{k l}<_{0} u_{i j}$. To prove that they are resolvable we need to prove that there exists composition of reductions $r$ and $r^{\prime}$ such that

$$
r L_{(12)}(x)=r^{\prime} L_{(23)}(x)
$$

where

$$
L_{(12)}(x)=L\left(u_{r s} u_{k l}\right) u_{i j}, \quad L_{(23)}(x)=u_{r s} L\left(u_{k l} u_{i j}\right) .
$$

We have to consider different cases. If $b(x)=0$,

$$
L_{(12)} L_{(23)} L_{(12)}(x)=L_{(23)} L_{(12)} L_{(23)}(x),
$$

i.e., everything moves at the price of a constant. We proceed similarly if $b(x)=$ 1 . The case $b(x)=3$ does not occur, since only pairs of the form $\left(a_{i j}, a_{k l}^{*}\right)$ satisfy the (A) relations, and pairs of the form $\left(a_{i j}, a_{k l}\right)$ are zero divisors or, if they are on the diagonal of $A$, they commute. Then either the relation holds trivially or it gives the case $b(x)=2$. We are thus left to discuss this case. Here

$$
x=\left\{\begin{array}{l}
a_{i j}^{*} a_{k l}^{*} a_{r s}, \\
a_{i j} a_{k l}^{*} a_{r s}^{*}, \\
a_{i j} a_{k l}^{*} a_{r s}, \\
a_{i j}^{*} a_{k l} a_{r s}, \\
a_{i j} a_{k l} a_{r s}^{*} .
\end{array}\right.
$$

If any pair of the form $\left(a_{i j}^{*}, a_{k l}^{*}\right)$ or $\left(a_{i j}, a_{k l}\right)$ is a zero divisor then it holds trivially. If it is not a zero divisor, i.e., we consider the diagonal entries of $A$, then we move one $a_{k l}^{*}$ or $a_{k l}$ and we are left with something that commutes and we are back to $b(x)=1$. Thus the result holds.

The following result then follows from this and [14].

Theorem 4.2. For any total ordering $<$ on the matrix elements $u_{i j}$ there exists a basis for $A_{q}(\mathrm{Sp}(k))$ consisting of

$$
\left\{u_{i_{1} j_{1}}^{r_{1}} \cdots u_{i_{m} j_{m}}^{r_{m}}: m=n^{2}, r_{i} \in \mathbb{Z}_{+}, u_{i_{1} j_{1}}<u_{i_{2} j_{2}}<\cdots<u_{i_{m} j_{m}}\right\} .
$$

Remark. The theorem yields a basis for every ordering.

Let us now specify the ordering. Let $N^{+}, N^{-}$be the subalgebras generated by the elements $u_{i j}, j<N+1-i$, and by $u_{i j}, j>N+1-i$, respectively. Let $H$ be the abelian subalgebra generated by the elements $u_{i, N+1-i} . H$ is generated by the elements on the antidiagonal of $u$, where $N^{+}$, respectively $N^{-}$, is generated by the elements above, respectively below, the antidiagonal of $u$. Then

$$
A_{q}(\operatorname{Sp}(k))=N^{+} \otimes_{\mathrm{C}} H \otimes_{\mathrm{C}} N^{-} .
$$

Now we want to construct representations of $A_{q}(\mathrm{Sp}(k))$ by using the Verma module construction. Let $G(N)$ be the wreath product of $\mathbb{Z} / 2$ by $S(k)$, where 
$S(k)$ denotes the permutation group on $k$ letters [15]. Let $\rho \in G(N)$ be arbitrary. Here $\rho$ represents the permutation and sign changes of $\{1, \ldots, k\}$, i.e., $\rho(-k)=-\rho(k)$. Set

$$
\begin{aligned}
& N_{\rho}^{-}=\left\{u_{\rho(i), j}: 1 \leq j<i<N\right\} \\
& H_{\rho}=\left\{u_{\rho(i), i}: 1 \leq i \leq N\right\} \\
& N_{\rho}^{+}=\left\{u_{\rho(i), j}: 1 \leq j, 1 \leq i<j \leq N\right\} \cup\{\text { zero divisors }\} .
\end{aligned}
$$

Choose an ordering on the matrix elements $u_{i j}$ such that all elements of $N_{\rho}^{-}$ are smaller than the elements of $H_{\rho}$ which are smaller than the elements of $N_{\rho}^{+}$. By $\mathscr{N}_{\rho}^{-}, \mathscr{H}_{\rho}, \mathscr{N}_{\rho}^{+}$we denote linear subspaces of $A_{q}(\mathrm{Sp}(k))$ generated by the basis elements, which by the theorem are products of elements of $\mathscr{N}_{\rho}^{-}$, $\mathscr{H}_{\rho}, \mathscr{N}_{\rho}^{+}$respectively, it follows that

$$
A_{q}(n)=\mathscr{N}_{\rho}^{-} \otimes_{\mathbb{C}} \mathscr{H}_{\rho} \otimes_{\mathbb{C}} \mathscr{N}_{\rho}^{+},
$$

in the sense that $n_{\rho}^{-} h_{\rho} n_{\rho}^{+}$yields a basis for $A_{q}(\mathrm{Sp}(k))$ whenever $n_{\rho}^{-} h_{\rho} n_{\rho}^{+}$runs through a basis of $\mathscr{N}_{\rho}^{-}, \mathscr{H}_{\rho}, \mathscr{N}_{\rho}^{+}$.

Let $L_{\rho}$ be the left ideal generated by the matrix elements of $N_{\rho}^{+}$. Then $L_{\rho}$ is the linear span of elements of the form $n_{\rho}^{-} h_{\rho} n_{\rho}^{+}$, with $n_{\rho}^{+} \neq I$. Let

$$
\begin{array}{r}
i<j \quad \text { if } \rho(i)>\rho(j) \text { and } u_{\rho(i), i} u_{\rho(j), j}=u_{\rho(j), j} u_{\rho(i), i} \\
\text { or } u_{\rho(i), i} u_{\rho(j), j}=0=u_{\rho(j), j} u_{\rho(i), i} .
\end{array}
$$

Let

$$
\begin{aligned}
i<j \quad \text { if } \rho(i)<\rho(j) & \text { and }\left[u_{\rho(i), i} u_{\rho(j), j}\right]=(q-1 / q) u_{\rho(i), j} u_{\rho(j), i} \\
& \text { or } u_{\rho(i), i} u_{\rho(j), j}=0=u_{\rho(j), j} u_{\rho(i), i} .
\end{aligned}
$$

Since

$$
u_{\rho(j), i} \in N_{\rho}^{+}
$$

we have

$$
u_{\rho(i), i} u_{\rho(j), j}=u_{\rho(j), j} u_{\rho(i), i} \text { modulo } L_{\rho},
$$

i.e., $u_{\rho(i), i}$ commute modulo $L_{\rho}$. Let $\Gamma=\left(\gamma_{1}, \ldots, \gamma_{k}\right) \in \mathbb{C}^{k}$ be arbitrary. Define the left ideal $F_{\rho}(\Gamma)$ generated by the matrix elements of $N_{\rho}^{+}$and by the elements $u_{\rho(i), i}-\gamma_{i} I$. Then $L_{\rho} \subset F_{\rho}(\Gamma), I \notin F_{\rho}(\Gamma)$, by using the basis.

We introduce the highest weight modules for an arbitrary element $\rho \in G(N)$ as follows. A left $A_{q}$-module $M$ is a $\rho$-highest weight module if the module is generated by a $\rho$-highest weight vector $m \in M$. In our case a $\rho$-highest weight vector $m \in M$ of weight $\Gamma=\left(\gamma_{1}, \ldots, \gamma_{N}\right)$ satisfies $u_{\rho(i), i} m=\gamma_{i} m$ and $u_{k l} m=0$ for $u_{k l} \in N_{\rho}^{+}$. We can define

$$
W^{\rho}(\Gamma)=A_{q}(\operatorname{Sp}(k)) / F_{\rho}(\Gamma) \text { and } v^{+}=I\left(\bmod F_{\rho}(\Gamma)\right) .
$$

Thus $W^{\rho}(\Gamma)$ is a left $A_{q}$-module by left multiplication. Thus $W^{\rho}(\Gamma)$ is a $\rho$ highest weight module for $A_{q}$, by construction, with $\rho$-highest weight vector $v^{+}$of weight $\Gamma$. 
Theorem 4.3. (i) If $M$ is any $\rho$-highest weight module with $\rho$-highest weight vector $m$ of weight $\Gamma$, then there exists a unique surjective $A_{q}$-equivariant linear map $\varphi: W^{\rho}(\Gamma) \rightarrow M$ so that $\varphi\left(v^{+}\right)=m$.

(ii) For $i=N, \ldots, \rho(1)+1$, we have $u_{i 1} v=0$, for all $v \in W^{\rho}(\Gamma)$.

(iii) If $\Gamma \in\left(\mathbb{C}^{*}\right)^{N}$, then $W^{\rho}(\Gamma)$ has a unique proper maximal submodule.

(iv) For every $\rho \in G(N)$ and for every $\Gamma \in\left(\mathbb{C}^{*}\right)^{N}$ a unique irreducible $\rho$ highest weight module $L^{\rho}(\Gamma)$ exists.

Proof. Part (i) is similar to Proposition 4.35 of [15]. Define a linear map

$$
\varphi\left(n_{\rho}^{-} v^{+}\right)=n_{\rho}^{-} m
$$

Then $\varphi$ is well defined and $\varphi$ is "equivariant",

$$
\varphi\left(u_{i j} n_{\rho}^{-} v^{+}\right)=u_{i j} \varphi\left(n_{\rho}^{-} v^{+}\right),
$$

by using the fact that $W^{\rho}(\Gamma)$ is a left $A_{q}$-module. Since $M \cong \mathscr{N}_{\rho}^{-} m$ as vector spaces, the map $\varphi$ is surjective. The uniqueness follows. Now $W^{\rho}(\Gamma) \rightarrow$ $\mathscr{N}_{\rho}^{-} v^{+}$, as vector spaces. We can consider the subspace of $W^{\rho}(\Gamma)$ generated by the elements of the form

$$
\prod_{j \leq k<l \leq n} u_{\rho(k), l}^{p_{k l}} v^{+}, \quad p_{k l} \in \mathbb{Z}_{+} .
$$

For $l \geq k \geq j, \rho(i) \geq \rho(j)$, and $i \geq j$ we have the following commutation relations:

$$
u_{\rho(i), j} u_{\rho(k), l}= \begin{cases}u_{\rho(k), l} u_{\rho(i), j} & \text { if } \rho(k)<\rho(i), \\ q u_{\rho(k), l} u_{\rho(i), j} & \text { if } \rho(k)=\rho(i), \\ u_{\rho(k), l} u_{\rho(i), j}+ & (q-1 / q) u_{\rho(i), l} u_{\rho(k), j} \\ 0 & \text { if } \rho(k)>\rho(i), \\ 0 & \text { if } u_{\rho(i), j} u_{\rho(k), l} \text { is a zero divisor. }\end{cases}
$$

It follows, for $\rho(i)>\rho(j), i>j$, that

$$
u_{\rho(i), j} \prod_{j \leq k<l \leq n} u_{\rho(k), l}^{p_{k l}} v^{+}=0 .
$$

Indeed if $\rho(i)$ is maximal with respect to $\rho(i)>\rho(j), i>j$, since $k>j$, then the third commutation relation of (4.2) does not occur and we have that (4.3) follows from the fact that $u_{\rho(i), j} v^{+}=0$.

To prove (4.3) for general $\rho(i)$, assume that it holds for all $u_{\rho(k), j}$ with $\rho(k)>\rho(j), k>j$. Then $\rho(k)>\rho(i)$. Use induction on $\sum p_{k l}=t$. Use of (4.2) gives the induction step. The third case may cause some problems but we use induction on $\rho(i)$, since $\rho(k)>\rho(i)>\rho(j)$, for $k \geq j$. Taking $j=1$, we get (ii). The zero divisors do not cause any problems since we get that (ii) holds trivially in such case. (iii) and (iv) follow from [14], [17]. By following the same argument of Proposition 3.5 of [14] we have that $L^{\rho}(\Gamma)$ is an irreducible highest weight module. To prove that $L^{\rho}(\Gamma)$ is an irreducible $\mathrm{Pol}\left(\mathrm{Sp}_{q}(\tau)\right)$ module, we need to consider what happens to the quantum determinant $D$. Set

$$
D^{-1} v=\left(\gamma_{1}, \ldots, \gamma_{k}\right)^{-1}(-q)^{l(\rho)} v, \quad \text { for every } v \in L^{\rho}(\Gamma),
$$


where $l(\rho)$ represents the length of the permutation $\rho$. Since $D$ is a central element it acts as scalar on $L^{\rho}(\Gamma)$, and the action on the highest weight vector $v^{+}$is given by

$$
D v^{+}=\sum_{\sigma \in S_{n}}(-q)^{l(\rho)} u_{\sigma(1), 1} \cdots u_{\sigma(N), N} v^{+},
$$

all the terms will cancel out except for $\rho=\sigma$, since $v^{+}$is a highest weight vector. Then the result holds and we conclude that $L^{\rho}(\Gamma)$ is irreducible.

\section{REFERENCES}

1. V. G. Drinfeld, Quantum groups, Proc. ICM Berkeley, 1986, pp. 798-820.

2. M. Jimbo, Introduction to the Yang-Baxter equation, Braid Group, Knot Theory and Statistical Mechanics (C. N. Yang and M. L. Ge, eds.), Adv. Ser. Math. Phys., vol. 9, World Sci. Publishing, Singapore, 1989, pp. 111-134.

3. S. L. Woronowicz, Compact matrix pseudogroups, Comm. Math. Phys. 111 (1987), 613-665.

4. S. Majid, Quasi triangular Hopf algebras and Yang-Baxter equations, Internat. J. Modern Phys. A 5 (1990), 1-91.

5. A. Paolucci, A new example of quantum groups, Ph.D. Thesis, University of Iowa, Iowa City, 1992.

6. __ An example of a quantum group: twisted $\mathrm{Sp}_{q}(2)$, Proc. Amer. Math. Soc. (to appear).

7. N. Yu. Reshetikhin, L. A. Takhtajan, and L. D. Faddeev, Quantification of Lie groups and Lie algebras, Algebra i Analiz. 1 (1989), 178-206. (Russian)

8. J. M. Vallin, $C^{*}$-algèbra de Hopf et $C^{*}$-algèbre de Kac, Proc. London Math. Soc. (3) 50 (1985), 131-174.

9. S. Baaj and G. Skandalis, Unitaires multiplicatifs et dualité pour les produits croisés de $C^{*}$-algèbres, version préliminaire.

10. V. Guillemin and A. Pollack, Differential topology, Prentice-Hall, Englewood Cliffs, NJ, 1974.

11. N. Yu. Reshetikhin, L. A. Takhtajan, and L. D. Faddeev, Quantum groups, Braid Group, Knot Theory and Statistical Mechanics (C. N. Yang and M. L. Ge, eds.), Adv. Ser. Math. Phys., vol. 9, World Sci. Publishing, Singapore, 1989, pp. 97-110.

12. S. L. Woronowicz, Twisted SU(2) group. An example of a non commutative differential calculus, Publ. Res. Inst. Math. Sci. 23 (1987), 117-181.

13. _ Tannaka-Krein duality for compact matrix pseudogroups. Twisted $\mathrm{SU}(N)$ groups, Invent. Math. 93 (1988), 35-76.

14. H. T. Koelink, On *-representations of the Hopf-algebra associated with the quantum group $U_{q}(N)$, Compositio Math. 77 (1991), 199-231.

15. A. W. Knapp, Representation theory of semisimple groups, Princeton Univ. Press, Princeton, NJ, 1986.

16. G. Bergman, The diamond lemma for ring theory, Adv. Math. 29 (1978), 178-218.

17. J. E. Humphreys, Introduction to Lie algebras and representation theory, Graduate Texts in Math., no. 9, Springer-Verlag, Berlin and New York, 1972.

Department of Mathematics, University of Iowa, Iowa City, lowa 52242 